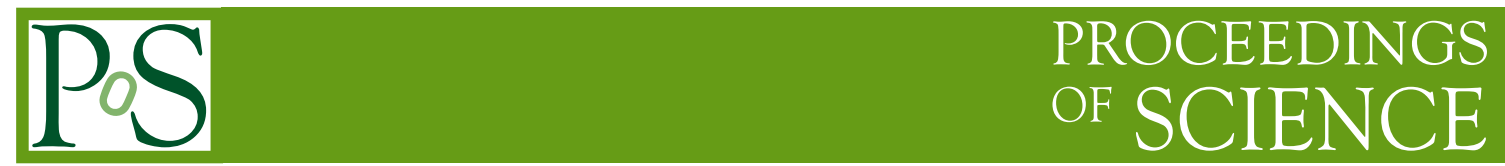

\title{
Charm Quarks and the QCD Equation of State
}

\section{Michael Cheng* for the RBC-Bielefeld Collaboration}

Department of Physics, Columbia University, New York, NY 10027, USA

E-mail: michaelc@phys.columbia.edu

\begin{abstract}
We present a study of the effect of charm quarks on the QCD equation of state using partiallyquenched $\mathrm{p} 4$ charm quarks on a dynamically generated $2+1$ flavor background, at zero chemical potential. We show preliminary results for the charm quark contribution to the energy density and pressure in the high temperature region $\left(T_{c}<T<4 T_{c}\right)$ and compare it to the free-field calculation. The charm quark mass is determined by measuring the charmonium spectrum.
\end{abstract}

The XXV International Symposium on Lattice Field Theory

July 30 - August 42007

Regensburg, Germany

${ }^{*}$ Speaker. 


\section{Introduction}

The equation of state(EoS) of QCD, i.e. thermodynamic quantities such as the pressure $(p)$, energy density $(\varepsilon)$, or entropy density $(s)$ as a function of the temperature, is important in understanding the high-temperature behavior of QCD. The EoS is not only of theoretical interest, but is directly applicable to the dynamics of the quark-gluon plasma (QGP), whether in the context of interpreting the results of heavy-ion experiments or modelling the behavior of hot, dense matter in the early universe.

Currently, some aspects of the EoS are accessible at high temperatures via perturbation theory [1], or at low temperatures via the hadron resonance gas model (HRG) [2]. However, a truly nonperturbative, first-principles calculation can only be done using lattice QCD.

Recently, there have been several detailed studies of the QCD EoS using improved actions and physical (or almost-physical) values for the light and strange quark masses $[3,4,5]$. While these studies accurately represent the three lightest quark flavors, they, like all previous calculations, neglect the effect of the charm quarks. However, recent work [6] based on perturbation theory indicates that the charm quark contribution may start to become significant at temperatures $T \sim 350$ $\mathrm{MeV}$, well within the temperature range covered by these latest EoS calculations.

In this work, we present a partially quenched study of the charm quark contribution to the QCD equation of state using p4fat 3 fermions on a $2+1 \mathrm{f}$ gauge background in the temperature range $0.90 T_{c}<T<4.2 T_{c}$. Currently, the calculations are done only for $N_{t}=4$ lattices, with the intention of continuing to $N_{t}=6$ and possibly $N_{t}=8$.

\section{Equation of State on the lattice}

We will first review the formalism for calculating the equation of state on the lattice via the "integral method", as described in [7]. First, consider the grand canonical partition function of QCD at vanishing quark chemical potential $\left(\mu_{q}=0\right)$ :

$$
Z(V, T)=\int\left[\mathscr{D} A_{\mu}\right][\mathscr{D} \Psi][\mathscr{D} \bar{\Psi}] \exp \left(-\int_{V} d^{3} x \int_{0}^{1 / T} d \tau \mathscr{L}_{Q C D}\right)
$$

We can extract various thermodynamics quantities from $\ln Z(T, V)$ such as the grand canonical potential $(\Omega(T, V))$, pressure $(p)$, or energy density $(\varepsilon)$ :

$$
\Omega(T, V)=T \ln Z(T, V) ; \varepsilon=\frac{E}{V}=-\frac{1}{V} \frac{\partial \ln Z}{\partial(1 / T)} ; p=T \frac{\partial \ln Z}{\partial V}
$$

In the thermodynamic limit, the grand canonical potential is an extensive quantity $(\Omega \sim V)$. Thus, we can write:

$$
p=\frac{T}{V} \ln Z(T, V) ; \varepsilon=\frac{T^{2}}{V} \frac{\partial \ln Z(V, T)}{\partial T}
$$

We can also define another quantity, called the interaction measure $I$, which is the trace of the energy-momentum tensor:

$$
\frac{I}{T^{4}}=\frac{\varepsilon-3 p}{T^{4}}=T \frac{\partial}{\partial T}\left(\frac{p}{T^{4}}\right)
$$


Unfortunately, calculating the pressure by computing $\ln Z(T, V)$ directly is not possible using stochastic Monte Carlo methods. However, we are able to compute certain derivatives of $\ln Z(T, V)$ which, in the lattice formulation, become the expectation values of operators. In particular, we can obtain the gauge action and the chiral condensate in this manner:

$$
\left\langle S_{g}\right\rangle=\frac{\partial \ln Z}{\partial \beta} ;\left\langle\bar{\psi} \psi_{q}\right\rangle=\frac{\partial \ln Z}{\partial \tilde{m}_{q}}
$$

where $\beta=6 / g_{0}^{2}$ is related to the bare coupling $g_{0}$ and $\tilde{m}_{q}$ denotes a bare quark mass.

On the lattice, finite temperatures can be simulated by limiting the temporal size of the lattice. This is directly analagous to restricting the integral in 2.1 to a finite interval in the imaginary time direction. The temperature is related to the temporal extent by $T^{-1}=N_{t} a\left(g_{0}, \tilde{m}_{q}\left(g_{0}\right)\right)$, where the lattice spacing $a$ is a function of both $g_{0}$ and $\tilde{m}_{q}$, and the bare quark masses $\tilde{m}_{q}$ must be adjusted as a function of $g_{0}$ along some renormalization group trajectory to keep physical quantities fixed, i.e. a line of constant physics.

We can rewrite the expression 2.4 for the interaction measure in terms of lattice quantities:

$$
\frac{I}{T^{4}}=\left(\frac{N_{t}}{N_{s}}\right)^{3}\left(\frac{\partial \beta}{\partial \ln a}\left(\left\langle S_{g}(T)\right\rangle-\left\langle S_{g}(0)\right\rangle\right)+\sum_{q} \frac{\partial \tilde{m}_{q}}{\partial \ln a}\left(\left\langle\bar{\psi} \psi_{q}(T)\right\rangle-\left\langle\bar{\psi} \psi_{q}(0)\right\rangle\right)\right)
$$

Here, the sum over the index $q$ indicates a sum over all quark flavors. We have also normalized the interaction measure by subtracting the $T=0$ contribution, disentangling the vacuum contribution from the thermal effects.

Using $I$, we can reconstruct other thermodynamic quantities. For example, we use 2.4 to express the pressure in terms of lattice quantities:

$$
\begin{aligned}
& \frac{P}{T^{4}}=\int_{0}^{T} \frac{I}{T^{\prime 4}} d \ln T^{\prime} \\
& \frac{P}{T^{4}}=\left(\frac{N_{t}}{N_{s}}\right)^{3} \int_{\beta_{0}}^{\beta}\left(\left(\left\langle S_{g}(T)\right\rangle-\left\langle S_{g}(0)\right\rangle\right)+\sum_{q} \frac{\partial \tilde{m}_{q}}{\partial \beta^{\prime}}\left(\left\langle\bar{\psi} \psi_{q}(T)\right\rangle-\left\langle\bar{\psi} \psi_{q}(0)\right\rangle\right)\right) d \beta^{\prime}
\end{aligned}
$$

Once we have $I(T)$ and $p(T)$, we can easily reconstruction the energy density, $\varepsilon(T)$, or the entropy density, $s(T)$. For a more detailed discussion of our EoS calculation with $2+1$ flavors, see [5].

\section{Calculation Method}

For this calculation, we are concerned only with the charm quark contribution. The relevant part of 2.6 is:

$$
\frac{I_{c}}{T^{4}}=\left(\frac{N_{t}}{N_{s}}\right)^{3} \frac{d \beta}{d \ln a} \frac{\partial \tilde{m}_{c}}{\partial \beta}\left(\left\langle\bar{\psi} \psi_{c}(T)\right\rangle-\left\langle\bar{\psi} \psi_{c}(0)\right\rangle\right)
$$

To calculate $\bar{\psi} \psi_{c}$, we make partially quenched measurements of the chiral condensate on previously generated $2+1$ f gauge configurations, both at finite temperature $\left(N_{t}=4\right)$ and zero temperature $\left(N_{t}=32\right)$. These dynamical configurations use the p4fat 3 fermion action [8], and a tree-level improved Symanzik gauge action. 
In order to determine the temperature of each ensemble, we have chosen to set the scale using the static quark potential parameter $r_{0} . r_{0}$ is defined as:

$$
\left(r^{2} \frac{d V_{q \bar{q}}(r)}{d r}\right)_{r=r_{0}}=1.65
$$

We can then convert to physical units by using $r_{0}=0.469(7) \mathrm{fm}$. [9]. Note that the bare quark masses $\left(\tilde{m}_{u d}\right.$ and $\tilde{m}_{s}$ ) are tuned as a function of $\beta$ so that physical quantities such as $m_{\pi} r_{0}, m_{\eta} r_{0}$, and $m_{K} r_{0}$ take on approximately constant values over the entire temperature range.

To determine $I_{c}$, we not only need $\bar{\psi} \psi_{c}$ on zero and finite temperature lattices, but also $d \beta / d \ln a$. We can deduce $d \beta / d \ln a$ from our scale-setting calculations of $r_{0}$ :

$$
\frac{d \beta}{d \ln a}=a \frac{d \beta}{d a}=\left(\frac{\partial \ln \left(a / r_{0}\right)}{\partial \beta}\right)^{-1}
$$

To get a smooth function for $d \beta / d \ln a$, we fit $\frac{a}{r_{0}}(\beta)$ to a renormalization-group inspired ansatz. Table 1 gives details of the input parameters for the lattices, the number of trajectories for the different ensembles, as well as $r_{0}$ and $T / T_{c}$. For more details about scale setting and the line of constant physics, see [5].

\begin{tabular}{|ccccccccr|}
\hline & & & & & & \multicolumn{3}{c|}{ Trajectories } \\
$\beta$ & Volume & $\tilde{m}_{u d}$ & $r_{0} / a$ & $T / T_{c}$ & $\tilde{m}_{c}\left(\eta_{c}\right)$ & $\tilde{m}_{c}(J / \Psi)$ & $N_{t}=32$ & $N_{t}=4$ \\
\hline 3.277 & $16^{3}$ & .00765 & $1.797(19)$ & 0.90 & 2.37 & 2.25 & 3250 & 12160 \\
3.335 & $16^{3}$ & .00570 & $2.033(17)$ & 1.06 & 1.57 & 1.42 & 2630 & 14280 \\
3.351 & $16^{3}$ & .00592 & $2.069(12)$ & 1.10 & 1.50 & 1.35 & 6950 & 12420 \\
3.382 & $16^{3}$ & .00520 & $2.225(13)$ & 1.20 & 1.22 & 1.11 & 2270 & 8110 \\
3.41 & $16^{3}$ & .00412 & $2.503(18)$ & 1.31 & 1.02 & .858 & 2790 & 16000 \\
3.46 & $16^{3}$ & .00313 & $2.890(16)$ & 1.50 & .670 & .650 & 2510 & 10200 \\
3.49 & $16^{3}$ & .00290 & $3.223(31)$ & 1.62 & .566 & .529 & 4290 & 9420 \\
3.51 & $16^{3}$ & .00259 & $3.423(61)$ & 1.70 & .508 & .473 & 2450 & 10000 \\
3.54 & $16^{3}$ & .00240 & $3.687(34)$ & 1.83 & .446 & .417 & 4060 & 6250 \\
3.57 & $24^{3}$ & .00212 & $4.009(26)$ & 1.98 & .386 & .347 & 2460 & 21190 \\
3.63 & $24^{3}$ & .00170 & $4.651(41)$ & 2.28 & .304 & .288 & 3290 & 10000 \\
3.69 & $24^{3}$ & .00150 & $5.201(48)$ & 2.61 & .257 & .244 & 2290 & 9470 \\
3.76 & $24^{3}$ & .00130 & $6.050(61)$ & 3.05 & .213 & .205 & 1110 & 33370 \\
3.82 & $24^{3}$ & .00110 & $6.752(96)$ & 3.46 & .181 & .190 & 3000 & 35000 \\
3.92 & $24^{3}$ & .00092 & $7.59(12)$ & 4.23 & .162 & .157 & 4080 & 35870 \\
\hline
\end{tabular}

Table 1: Input parameters for the ensembles on which we have performed measurements. Note, $\tilde{m}_{s}=10 \tilde{m}_{u d}$ for all of these ensembles. Also given are $r_{0}, T / T_{c}, \tilde{m}_{c}$ determined from $m_{\eta_{c}}$ and $m_{J / \Psi}$, and the number of trajectories for $N_{t}=4$ and $N_{t}=32$.

\section{Setting the physical charm mass}

In addition to $d \beta / d \ln a$, we also need $\partial \tilde{m}_{c} / \partial \beta$ to calculate $I_{c}$. Thus, we need some method to determine the bare charm quark mass so that it also sits on a line of constant physics as we vary $\beta$. 

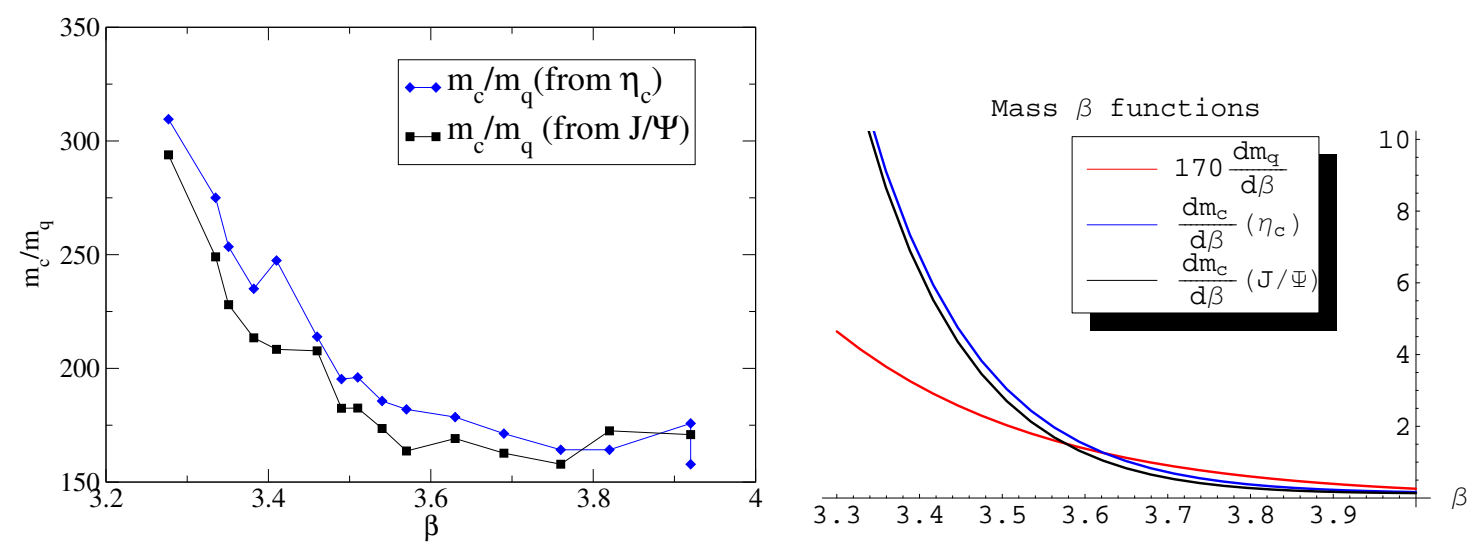

Figure 1: On the left, we see $\tilde{m}_{c} / \tilde{m}_{u d}$ as a function of $\beta$. On the right, we see $\partial \tilde{m}_{c} / \partial \beta$.

Ideally, if we were simulating in the scaling regime for charm quark observables, the ratio $\tilde{m}_{c} / \tilde{m}_{u d}$ should be a constant throughout the entire temperature range and independent of the quantity chosen to fix $\tilde{m}_{c}$. Unfortunately, $\tilde{m}_{c}$ is quite large on the available lattices, so the ratio $\tilde{m}_{c} / \tilde{m}_{u d}$ depends on $\beta$ and the particular quantity used to set $\tilde{m}_{c}$.

In order to understand the extent of this scaling violation, and how it affects our calculation, we have chosen to fix $\tilde{m}_{c}$ by setting two different charmonium states to their physical masses $\left(m_{\eta_{c}}\right.$ $=2.980 \mathrm{GeV}$ and $m_{J / \Psi}=3.097 \mathrm{GeV}$ ). This produces a range of values $0.16<\tilde{m}_{c}<2.4$, with exact figures given in Table 1 . As seen in Figure 1, we find fairly reasonable scaling $\left(\tilde{m}_{c} / \tilde{m}_{u d} \sim\right.$ $170)$ for the finer lattice spacings $\left(\beta>3.5 \rightarrow T>1.7 T_{c}\right)$. However, as we lower $\beta$ onto coarser lattices, we find that this approximate scaling breaks down, and this ratio $\tilde{m}_{c} / \tilde{m}_{u d}$ begins to increase dramatically. We also find that using the $\eta_{c}$ state tends to give a systematically higher value for $\tilde{m}_{c}$, although this ultimately does not have a large affect on the final calculation.

Once we have $\tilde{m}_{c}(\beta)$, we can fit this data to a RG-inspired ansatz for the running of the bare quark mass. This allows us to calculate a smooth curve for $\partial \tilde{m}_{c} / \partial \beta$, also shown in Figure 1 . We see that this $\beta$-function increase s dramatically as we move to smaller $\beta$, in contrast to the scaled version of $\partial \tilde{m}_{u d} / \partial \beta$, which shows a much milder $\beta$-dependence.

\section{Results}

Figure 2 shows our results for the charm contribution using both $\eta_{c}$ and $J / \Psi$ to determine $\tilde{m}_{c}$, as well as a free-field calculation using the physical value of the charm mass $m_{c} \approx 1.2 \mathrm{GeV}$. The choice of $\eta_{c}$ or $J / \Psi$ does have some effect, but both curves share the same qualitative features. Notably, the interaction measure $I_{c}$ increases drastically as we move through the transition. This is consistent with the notion that the charmonium states are the lightest charm states that contribute below the transition, and thus are exponentially suppressed by their heavy mass.

It is interesting to note that the value of $I_{c}$ already becomes quite large even just above the transition $\left(T \sim 1.1 T_{c}\right)$, decaying away slowly as the temperature is increased. This is in contrast to the free-field calculation, where the interaction measure peaks at $T \sim 0.35 m_{c} \approx 400 \mathrm{MeV}$, although of course the free-field result knows nothing about the crossover transition. Perhaps more surprising is the fact that $I_{c}$ is significantly greater than the free-field value for $T<3 T_{c}$. As a result, $p$ and $\varepsilon$ 

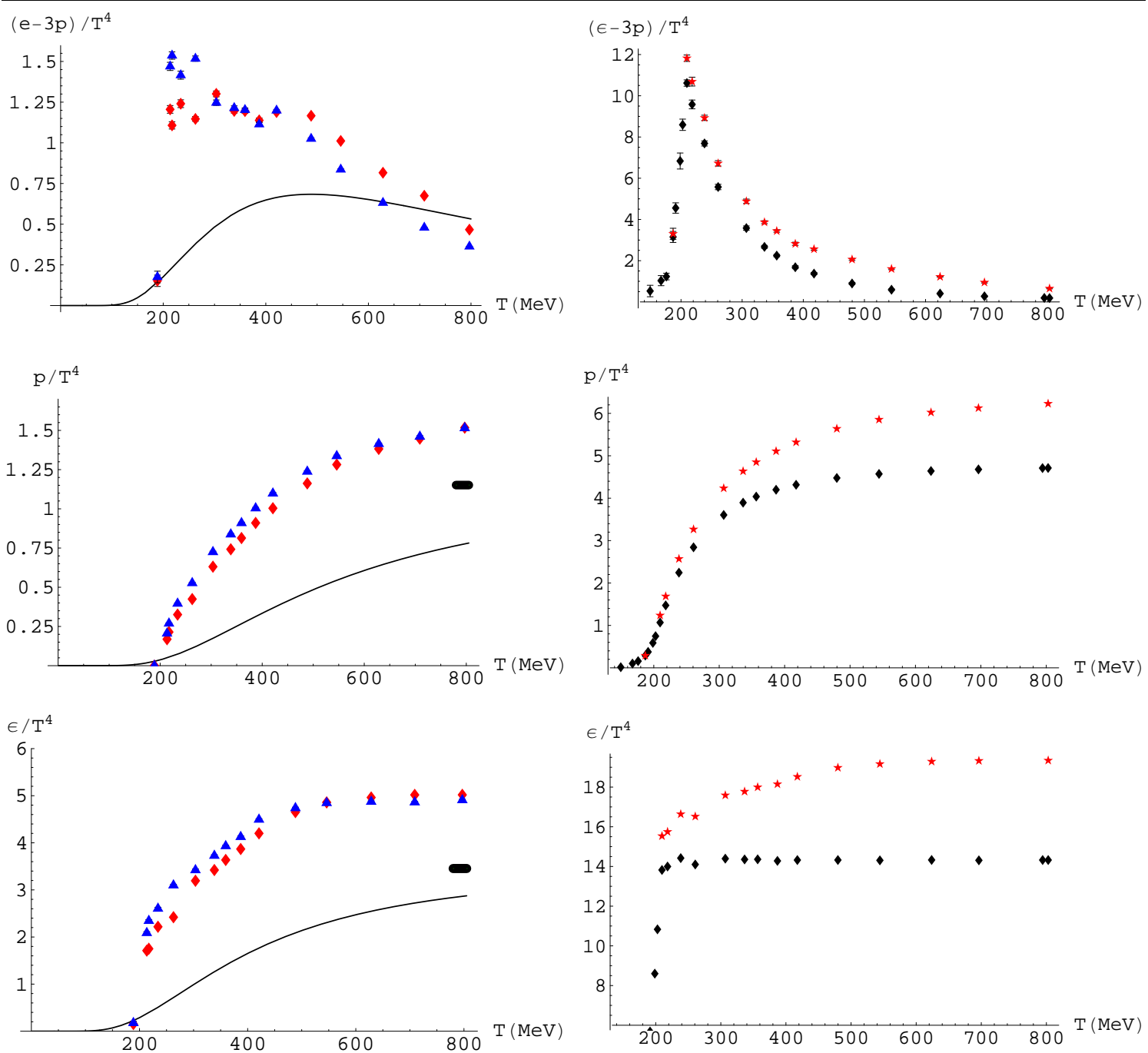

Figure 2: In the left column, the charm contribution to the interaction measure $\left(\frac{\varepsilon-3 p}{T^{4}}\right)$, pressure $\left(\frac{p}{T^{4}}\right)$, and energy density $\left(\frac{\varepsilon}{T^{4}}\right)$, respectively. Diamonds use $\eta_{c}$ while the triangles use $J / \Psi$ to set $\tilde{m}_{c}$. The solid curve gives the free-field results, while the solid bars on the upper right give the $T \rightarrow \infty$ limit. In the right column, the charm contribution (stars) is added onto the pure $2+1 \mathrm{f}$ part (diamonds).

increase much faster just above the transition than expected. This causes $p$ and $\varepsilon$ to overshoot their continuum Stefan-Boltzmann values $T \rightarrow \infty$ by a large amount, even at finite temperature. This difference may be attributed to finite lattice spacing corrections at $N_{t}=4$. Indeed, for the $2+1 \mathrm{f}$ calculation, the contribution of the quark condensates to $(\varepsilon-3 p) / T^{4}$ decreases going from $N_{t}=4$ to $N_{t}=6$.

Figure 2 also shows the charm contribution superimposed on the $2+1$ f calculation at $N_{t}=4$. Although we see little change in the interaction measure because of the high $2+1 \mathrm{f}$ peak, the pressure and energy change by quite a large amount for $T>T_{c}$.

\section{Conclusion}

We have made a partially quenched calculation of the charm quark equation of state. Current EoS studies are in a temperature regime that is a significant fraction of the charm quark mass, 
where the dynamics of the charm quark may have a significant effect and can no longer be ignored.

Admittedly, this calculation has unquantified, possibly large systematic errors. First of all, our calculations are partially quenched, so that the full dynamics of the charm quark may not be accurately reflected in their "back-reaction" on the gauge fields. To the extent that the charm quark is heavy, this should be a small effect, but may be problematic at higher temperatures and at finer lattice spacings. Secondly, $\tilde{m}_{c}$ is a large fraction of, or even exceeds, the lattice spacing on many of the lattices on which we measure. This introduces large cut-off effects, and it is appropriate to ask whether the $\mathrm{p} 4$ fermion formulation can give even a reasonable facsimile of the charm quark dynamics at these heavy masses.

As there are no plans for large-scale simulations involving a dynamical charm quark, it seems we must tolerate the first problem. The second problem may be alleviated somewhat be moving to finer lattice spacings, $\left(N_{t}=6,8\right)$. This may give us a better grasp of the discretization errors in the current calculation. Furthermore, the recent development of the HISQ action [10], which, among other improvements, removes the leading order $O(m a)^{4}$ corrections to the dispersion relation at tree-level, provides another possible tool to mitigate the large cut-off effects currently present.

The future plans for this calculation is extension to $N_{t}=6$ and possibly $N_{t}=8$, as well as investigating the possibility of adapting the methods used by the HISQ action. Hopefully, this will allow us to better understand why the $N_{t}=4$ calculation so badly overshoots the Stefan-Boltzmann limit, and to better quantify the cut-off effects.

\section{Acknowledgments}

This work was carried out in collaboration with the RBC-Bielefeld Collaboration. In particular we thank Norman Christ, Frithjof Karsch, and Peter Petreczky for useful discussions. Computations were performed on the RIKEN-BNL QCDOC machines at Brookhaven National Laboratory. We thank RIKEN, BNL, Columbia University, and the US DOE for providing the facilities on which this work was done. This research was supported by US DOE grants DE-AC02-98CH1-886 and DE-FG02-92ER40699.

\section{References}

[1] A. Vuorinen, Phys. Rev. D68, (2003) 054017, [hep-ph/0305183].

[2] P. Braun-Munzinger, K.Redlich, and J. Stachel (2003), nucl-th/0304013.

[3] C. Bernard et al., Phys. Rev. D75, (2007) 094505, [hep-lat/0611031].

[4] Y. Aoki, Z. Fodor, S. D. Katz, and K. K. Szabo, JHEP 01, (2006) 089, [hep-lat/0510084].

[5] M. Cheng et. al., (2007), hep-lat/0710.0354.

[6] M. Laine and Y. Schroder, Phys. Rev. D73, (2006) 085009, [hep-ph/0603048].

[7] C. Bernard et. al. (MILC), Phys. Rev. D55, (1997) 6861, [hep-lat/9612025].

[8] F. Karsch, E. Laermann, and A. Peikert, Phys. Lett. B478, (2000) 447, [hep-lat0002003].

[9] A. Gray et. al., phys. Rev. D72, (2005) 094507, [hep-lat/0507013].

[10] E. Follana et. al. (HPQCD), Phys. Rev. D75, (2007) 054502, [hep-lat/0610092]. 